\title{
Diet and Inflammatory Bowel Disease: Thoughts on Food, Perceptions and Beliefs
}

\author{
Benjamin Crooks ${ }^{a, b} \quad$ John McLaughlin ${ }^{a-c}$ Jimmy K. Limdi ${ }^{c, d}$ \\ a Division of Diabetes, Endocrinology and Gastroenterology, University of Manchester, Manchester, UK; \\ ${ }^{b}$ Department of Gastroenterology, Salford Royal NHS Foundation Trust, Salford, UK; ' ${ }^{\top}$ Manchester Academic Health \\ Science Centre, Manchester, UK; d Division of Gastroenterology, The Pennine Acute Hospitals NHS Trust, Section of \\ Inflammatory Bowel Disease, Manchester, UK
}

Dear Editor,

We read with interest the paper by de Vries et al. [1] reporting dietary beliefs and behaviours in a Dutch cohort with inflammatory bowel disease (IBD). Their findings corroborate with our observations from the United Kingdom and others [2, 3], particularly with certain food groups exacerbating symptoms such as spicy and fatty foods, fruits and vegetables and dairy products. This raises important questions that should inform and stimulate further research.

The authors acknowledge selection bias from a national IBD patient group, a large proportion in self-reported disease remission, using dietary supplements and engaging in exercise and relaxation techniques to control IBD symptoms. The large proportion of patients in self-reported remission raises the possibility of symptoms being driven by other putative mechanisms such as psychosocial factors, neuroendocrine influences including dysregulated satiety signalling in the brain-gut axis, or chemostimulation of gut receptors [4]. For example, the observation that patients noted carbohydrate-rich foods, fruits and cabbage among exacerbating factors may simply relate to FODMAP intake with their subsequent osmotic effect and fermentation by gut microbiota, independent of intestinal inflammation [4]. Likewise, high fat diets may trigger or exacerbate inflammation through diverse mechanisms including their effect on cytokine signalling, dysbiosis and intestinal permeability or by a direct effect on immune cells and toll-like receptors, associated with a low-grade inflammatory state even in healthy individuals [4].

Their striking observation that certain foods (cabbage and legumes) detrimental to some patients were considered beneficial by others is worthy of further study. It is plausible that studies on the microbiome, metabolomics and indeed nutrigenomics may contribute mechanistic insights into the nocebo and anti-inflammatory effects of certain food groups on the microbiota and immune system [5].

Dietary research is fraught with challenges, not in the least with capturing the type and proportion of food intake, the complex interactions between food groups, varied metabolism among individuals but also complexities with food handling, processing and packaging. Difficulties with disentangling the biology of food, the microbiome and the immune system not- withstanding, future studies should also assess food intake and effects in different IBD phenotypes, in active disease and in remission. Meanwhile, de Vries et al. [1] are to be commended for adding to our body of knowledge and raising as many important questions as they attempt to answer. The wider research community needs to add more scientific credibility for our observations in multiple settings before an evidence-based approach can be used to inform patients. More thought with food.

\section{Acknowledgements}

None.

\section{Disclosure Statement}

The authors declare that they have no conflicts of interest to disclose.

\section{Funding Sources}

No funding was received for writing this manuscript.
Benjamin Crooks

Division of Diabetes, Endocrinology and Gastroenterology, University of Manchester Ward 19 (Clinical Research Unit) Fairfield General Hospital

Rochdale Old Road Bury, Manchester BL9 7TD (UK)

E-Mail Benjamin.Crooks@ postgrad.manchester.ac.uk 


\section{References}

1 de Vries JH, Dijkhuizen M, Tap P, Witteman BJ. Patient's Dietary Beliefs and Behaviours in Inflammatory Bowel Disease. Dig Dis. 2019; 37(2):131-9.

2 Limdi JK, Aggarwal D, McLaughlin JT. Dietary Practices and Beliefs in Patients with Inflammatory Bowel Disease. Inflamm Bowel Dis. 2016 Jan;22(1):164-70.
3 Principi M, Losurdo G, Iannone A, Contaldo A, Deflorio V, Ranaldo N, et al. Differences in dietary habits between patients with inflammatory bowel disease in clinical remission and a healthy population. Ann Gastroenterol. 2018 Jul-Aug;31(4):469-73.

4 Khalili H, Chan SS, Lochhead P, Ananthakrishnan AN, Hart AR, Chan AT. The role of diet in the aetiopathogenesis of inflammatory bowel disease. Nat Rev Gastroenterol Hepatol. 2018 Sep;15(9):525-35.

5 Marlow G, Ellett S, Ferguson IR, Zhu S, Karunasinghe N, Jesuthasan AC, et al. Transcriptomics to study the effect of a Mediterranean-inspired diet on inflammation in Crohn's disease patients. Hum Genomics. 2013 Nov;7(1):24. 